\title{
Multimodal navigation in the functional microsurgical resection of intrinsic brain tumors located in eloquent motor areas: role of tractography
}

\author{
José M. González-Darder, Ph.D., ${ }^{1}$ Pablo González-LóPez, M.D., ${ }^{1}$ \\ Fernando Talamantes, M.D., ${ }^{1}$ Vicent Quilis, M.D., ${ }^{1}$ Victoria Cortés, M.D., 2 \\ Guillermo García-March, M.D., ${ }^{1}$ and Pedro Roldán, Ph.D. ${ }^{1}$
}

Departments of ${ }^{I}$ Neurosurgery and ${ }^{2}$ Neurophysiology, Hospital Clínico Universitario, Servicio Valenciano de Salud, Valencia, Spain

\begin{abstract}
Object. Nowadays the role of microsurgical management of intrinsic brain tumors is to maximize the volumetric resection of the tumoral tissue, minimizing the postoperative morbidity. The purpose of this paper was to study the benefits of an original protocol developed for the microsurgical treatment of tumors located in eloquent motor areas where the navigation and electrical stimulation of motor subcortical pathways have been implemented.

Methods. A total of 17 patients who underwent resection of cortical or subcortical tumors in motor areas have been included in the series. The preoperative planning for multimodal navigation was done by integrating anatomical studies, motor functional MR (fMR) imaging, and subcortical pathway volumes generated by diffusion tensor (DT) imaging. Intraoperative neuromonitoring included motor mapping by direct cortical stimulation (CS) and subcortical stimulation (sCS), and localization of the central sulcus by using cortical multipolar electrodes and the N20 wave inversion technique. The location of all cortically and subcortically stimulated points with positive motor response was stored in the navigator and correlated with the cortical and subcortical motor functional structures defined preoperatively.

Results. The mean tumoral volumetric resection was $89.1 \pm 14.2 \%$ of the preoperative volume, with a total resection $(\geq 100 \%)$ in 8 patients. Preoperatively a total of $58.8 \%$ of the patients had some kind of motor neurological deficit, increasing 24 hours after surgery to $70.6 \%$ and decreasing to $47.1 \%$ at 1 month later. There was a great correlation between anatomical and functional data, both cortically and subcortically. A total of 52 cortical points submitted to CS had positive motor response, with a positive correlation of $83.7 \%$. Also, a total of 55 subcortical points had positive motor response; in these cases the mean distance from the stimulated point to the subcortical tract was $7.3 \pm 3.1 \mathrm{~mm}$.

Conclusions. The integration of anatomical and functional studies allows a safe functional resection of the brain tumors located in eloquent areas. Multimodal navigation allows integration and correlation among preoperative and intraoperative anatomical and functional data. Cortical motor functional areas are anatomically and functionally located preoperatively thanks to MR and fMR imaging and subcortical motor pathways with DT imaging and tractography. Intraoperative confirmation is done with CS and N20 inversion wave for cortical structures and with sCS for subcortical pathways. With this protocol the authors achieved a good volumetric resection in cortical and subcortical tumors located in eloquent motor areas, with an increase in the incidence of neurological deficits in the immediate postoperative period that significantly decreased 1 month later. Ongoing studies must define the safe limits for functional resection, taking into account the intraoperative brain shift. Finally, it must be demonstrated whether this protocol has any long-term benefit for patients by prolonging the disease-free interval, the time to recurrence, or the survival time. (DOI: 10.3171/2009.11.FOCUS09234)
\end{abstract}

\begin{tabular}{llcl} 
KEY WORDS & \multicolumn{2}{c}{ brain tumor surgery $\bullet \quad$ cortical mapping } \\
subcortical mapping & $\bullet$ & intraoperative stimulation mapping \\
intrinsic brain tumor & $\bullet$ & tractography navigation
\end{tabular}

$\mathrm{T}$

HE role of radical surgery in the management of glial-type brain tumors is still controversial. In fact, there is no scientific evidence that a greater extent of

Abbreviations used in this paper: $\mathrm{CS}=$ cortical stimulation; $\mathrm{DT}=$ diffusion tensor; $\mathrm{fMR}=$ functional $\mathrm{MR} ; \mathrm{sCS}=$ subcortical stimulation; SSEP = somatosensory evoked potential.

resection is associated with a better prognosis. , $^{219,20,31,35,37}$ It does seem clear that the infiltrative growth pattern of the neuropil by glial tumors is the reason for surgical treatment not being curative, ${ }^{7}$ and therefore the majority of patients will suffer a relapse or local progression of the disease after surgery. The known molecular mechanisms that drive this pattern of cellular migration have 
also not allowed for the development of really effective treatments. ${ }^{11-13,16}$

Until new treatments for glial tumors are developed, the cytoreductive treatment provided by surgery will maintain its value, because it provides material for diagnosis and research, alleviates both the focal and clinical symptoms of intracranial hypertension, and contributes to a greater efficacy of oncological treatments. Following this line of reasoning, there is a large number of modern papers supporting the idea of resecting the largest possible volume of tumor. ${ }^{11,25,26,35}$ The price to pay for radical resection may be an increase in morbidity. In this sense, a great number of imaging, neurophysiological, neurochemical, and even surgical techniques have recently been developed and incorporated into the management of these tumors, with the ultimate objective being to optimize the resection limits; to extend them to the maximum while minimizing the eventual associated morbidity. ${ }^{32,33,38,39}$ The final objective of this approach is to resect the lesion identified as tumoral based on the imaging techniques, as well as the neighboring brain tissue that is normal in appearance during the surgery, but that is suspected of already being invaded by the tumor cells, or with cells already affected by the molecular disorder that would convert them into tumor cells. In this way, the "tumoral resection limit" would be taken to the point where there may be deterioration of any neurological function considered important ("functional resection limit").

The motor and sensory cortex, as well as their corticospinal and associated projections, are probably some of the most eloquent brain structures. For this reason, these are the most anatomically and functionally well-defined areas, but it is also true that they often become distorted by the tumor that is located in the area and is the reason for the surgical treatment. ${ }^{4,29,30}$

The objective of this work was to study the value of the new anatomical and functional pre- and intraoperative techniques in defining the functional resection limits in a series of tumors located in eloquent motor areas and undergoing microsurgical treatment. In accordance with this goal, a pre- and intraoperative study protocol has been designed that combines cortical anatomical localization techniques (CT scanning and different sequences of MR imaging) and functional localization techniques (fMR imaging and direct CS) as well as subcortical anatomical (DT imaging) and functional localization techniques (direct $\mathrm{SCS}$ ). These techniques are intraoperatively integrated and correlated with the aid of the navigation system. The ultimate objective of the study was to define an ideal protocol for the most radical and safest microsurgical management of tumors in eloquent motor areas, by studying the role of subcortical localization techniques.

\section{Methods}

The study was performed in a series of patients who underwent surgery for cortical or subcortical intrinsic brain tumors located within or close to eloquent motor areas. The following have been anatomically considered as eloquent motor areas. 1) In the frontal lobe, the precentral or vertical gyrus (primary motor area-Brodmann area 4), the posterior region of the frontal superior gyrus (premotor area-Brodmann area 6), the mesial region of the frontal superior gyrus (supplementary motor areaBrodmann area 8), and the inferoposterior part of the pars opercularis or frontal operculum (primary motor area and Broca area-Brodmann areas 44, 6, and 4). 2) In the parietal lobe, an important role is played by the postcentral gyrus (somatosensory area-Brodmann areas 3, 2, and 1) in the control of movement. 3) In the insula, the insular cortex is considered an important region in motor planning and motor speech (Brodmann area 13). 4) Finally, the bundle of somatosensory and associated motor tracts that form the internal capsule in the layer of the corona radiata is considered to be an eloquent area. ${ }^{3,34}$

All cases were studied prospectively, following the same basic clinical study protocol for imaging, surgical procedure, and postoperative controls. In all cases, informed consent was obtained from the patients for the different study, monitoring, and treatment techniques. This study has been approved by the ethics committee of the Hospital Clínico Universitario of Valencia, with a specific informed consent having been drawn up for inclusion in the study.

\section{Preoperative Study}

The clinical variables collected included age, sex, clinical history, symptoms and signs on diagnosis, and response to treatment at the time of surgery. The imaging studies for the initial diagnosis included a cranial CT scan without contrast, followed by an MR imaging study with an original protocol for tumor and morphological characterization. This protocol includes the following sequences: axial 3D T1-weighted, sagittal 3D magnetization-prepared rapid acquisition gradient echo, axial and coronal T2-weighted, axial FLAIR, perfusion, diffusion, spectroscopy, and axial T1-weighted imaging obtained with Gd contrast.

After the suspicion of a tumor was raised by the imaging study, a 3D volumetric reconstruction of the lesion was undertaken on a Medtronic Stealth planning station with the aid of the StealthViz software package, for which axial T1-weighted images with Gd were used in the lesions with enhancement, and axial FLAIR images were used in the lesions without evident contrast enhancement. The reconstruction had two objectives; the first was to determine the tumor volume, and the second was to confirm the location of the lesion in an eloquent motor area. For the second objective, the central sulcus was used as the anatomical reference, identified directly or with the aid of the ascending cingulate sulcus visible in the midline sagittal image, defining from this point the postcentral gyrus, precentral gyrus, superior frontal gyrus (anterior and posterior), insula, and frontal operculi. . $^{3,6,9,34}$ After the identification of these anatomical areas, the degree of eloquence was determined using the classification proposed by Sawaya et al., ${ }^{36}$ which classifies the lesions on the basis of their location relative to brain function as follows: Grade I, lesion in a noneloquent area; Grade II, lesion close to an eloquent area; and Grade III, lesion in an eloquent area. With the aim of preoperatively validating the real functional responsibility of these cortical anatomical areas considered as eloquent, an fMR imaging study was 


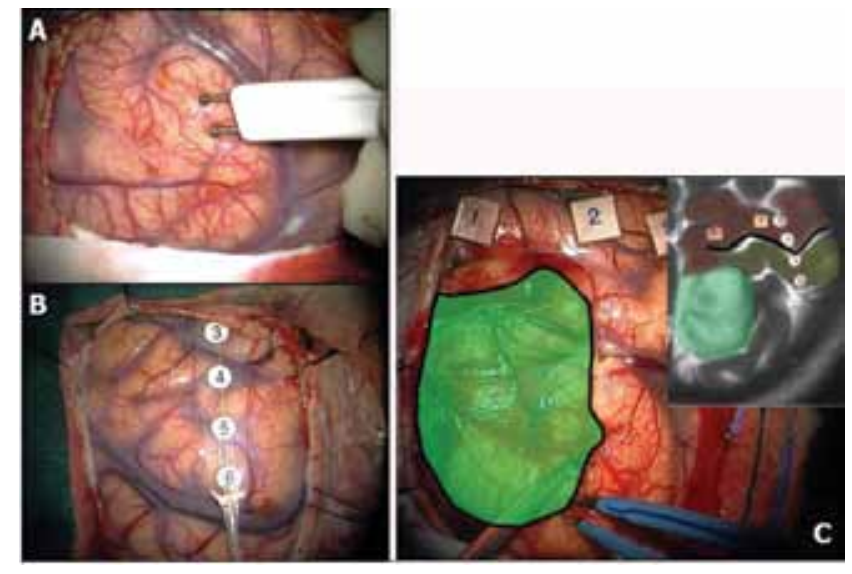

FIG. 1. Intraoperative views obtained during multimodal cortical navigation. View through the operating microscope after dura mater opening during mapping with CS (A) and placement of a flat electrode grid for localization of central sulcus (B). View through the operating microscope after opening of dura mater $(\mathbf{C})$ and T2-weighted MR image (inset) in navigator; green area contours the tumor, and the position of the central sulcus is marked by a black line.

performed with study paradigms of motor function (foot, hand, and tongue), superimposed over an anatomical MR imaging study. $1,3,14,17,21,27$

The preoperative evaluation was completed with a DT MR imaging study, and a tractography study of the tracts involved in each case was undertaken, especially the corticospinal pathway and superior longitudinal fasciculus. ${ }^{9}$ Thanks to a series of freely available software tools (MRIcro, dTV.II SR Volume One, MedINRIA, and Image J) the chosen tract was converted into volumes, which were superimposed over the 3D magnetization-prepared rapid acquisition gradient echo anatomical study, according to the pattern of uptake. The volumetric transformation of these tracts and their fusion with the anatomical studies was achieved in all cases, with a specific pattern of shades of gray to avoid confusion with the rest of the structures in the studies over which they were fused.

All the MR imaging studies were performed at the installations of Exploraciones Radiológicas Especiales SA in the Hospital Clínico Universitario of Valencia, and using the Siemens MAGNETOM Trio 3-T system and Signa Excite 3-T system (General Electric Healthcare).

\section{Fusion and Preoperative Planning}

Thanks to a network connection between the MR systems, the planning station, and the intraoperative navigator unit, all the imaging studies mentioned were sent directly from the study equipment to the aforementioned planning station situated in the Neurosurgery Department, where the fusion and posterior planning were completed. Depending on the pattern of enhancement, the 3D T1-weighted study with Gd contrast or the FLAIR study was chosen as the base for the fusion, over which the fMR imaging study and the volumes of the tracts generated from the tractography were fused. After the fusion, the preoperative planning was performed, and sent via the hospital's intranet directly to the navigator unit situated in the Department of Neurosurgery's operating room.

\section{Navigation and Intraoperative Neurophysiological} Monitoring

With the brain cortex exposed after craniotomy, the cortical projection of the lesion was located and the anatomical structures were identified with the navigator's probe by using the electromagnetic module. The routine neurophysiological monitoring was composed of 3 techniques. Immediately after the craniotomy and the anatomical localization of the structures with the navigator, cortical mapping was performed with CS (Nihon Kohden Neuropack equipment and Integra NeuroSciences Ojemann Cortical Stimulator [OCS-2]), identifying the cortical areas with a motor response, which were identified in the surgical field with marks (CS enabled mapping of the cortical motor area via direct bipolar stimulation and electromyographic recording) (Figs. 1 and 2).

In case of lesions around the central sulcus, this was identified with a 6-contact flat electrode and the N20 wave inversion technique (mapping of the central sulcus with SSEPs from the contralateral median nerve). To identify the central sulcus, we used the following registration and stimulation parameters: sensibility, 20-50 $\mu \mathrm{V}$; sweeping time, $5 \mathrm{msec} /$ division; filters, $1.5 \mathrm{kHz}-5 \mathrm{~Hz}$; pulse rate, $0.2 \mathrm{msec}$; square wave, $5.8 \mathrm{~Hz}$; and intensity between 20 and $25 \mathrm{~mA}$.

Finally, after the volumetric resection of the lesion based on the position of the subcortical tracts (volumes generated by the tractography), direct stimulation of the subcortical tracts was performed (sCS enabled subcortical motor mapping via direct bipolar stimulation and electromyographic recording). For CS and sCS we used the same stimulation parameters, which were a $60-\mathrm{Hz}$ square wave, 0.5 - to 1-msec pulse duration, during at least 2- to 4-second stimuli, and an ascendent intensity from 2 to $10 \mathrm{~mA}$, depending on the response.

As a control, transcranial stimulation of the ipsi- and contralateral motor cortex was performed with electromyographic recording of ipsi- and contralateral cortical SSEPs (Digitimer D185 Stimulator and Neuropack equipment). For this registration we used 4-7 pulse trains with stimuli duration of $50 \mu \mathrm{sec}, 150-200 \mathrm{~V}$ intensity, and a stimulus-free interval of $4 \mathrm{msec}$.

The combination of the anatomical information provided by navigation and the functional information provided by cortical mapping with CS allowed for the design of the transsulcal approach or, if applicable, the corticectomy. The anatomical information on the calculated volumetric resection provided by the navigator, along with the result (if applicable) from the mapping of the white matter with sCS, marked the resection limit. In both situations, however, the final decision on accepting the result of the planning or, in case of conflict, the pertinent modifications, was the ultimate responsibility of the surgeon in charge of the procedure.

The position of all the points where a positive CS or sCS motor response was obtained were marked with the probe, with the aim of analyzing the correlation between the stimulation, cortical and sulcal anatomical references, anatomical tract, and functional cortical area identified on the fMR imaging studies. In the sCS, the stimulation parameters (potential, intensity, and duration of stimulus) 

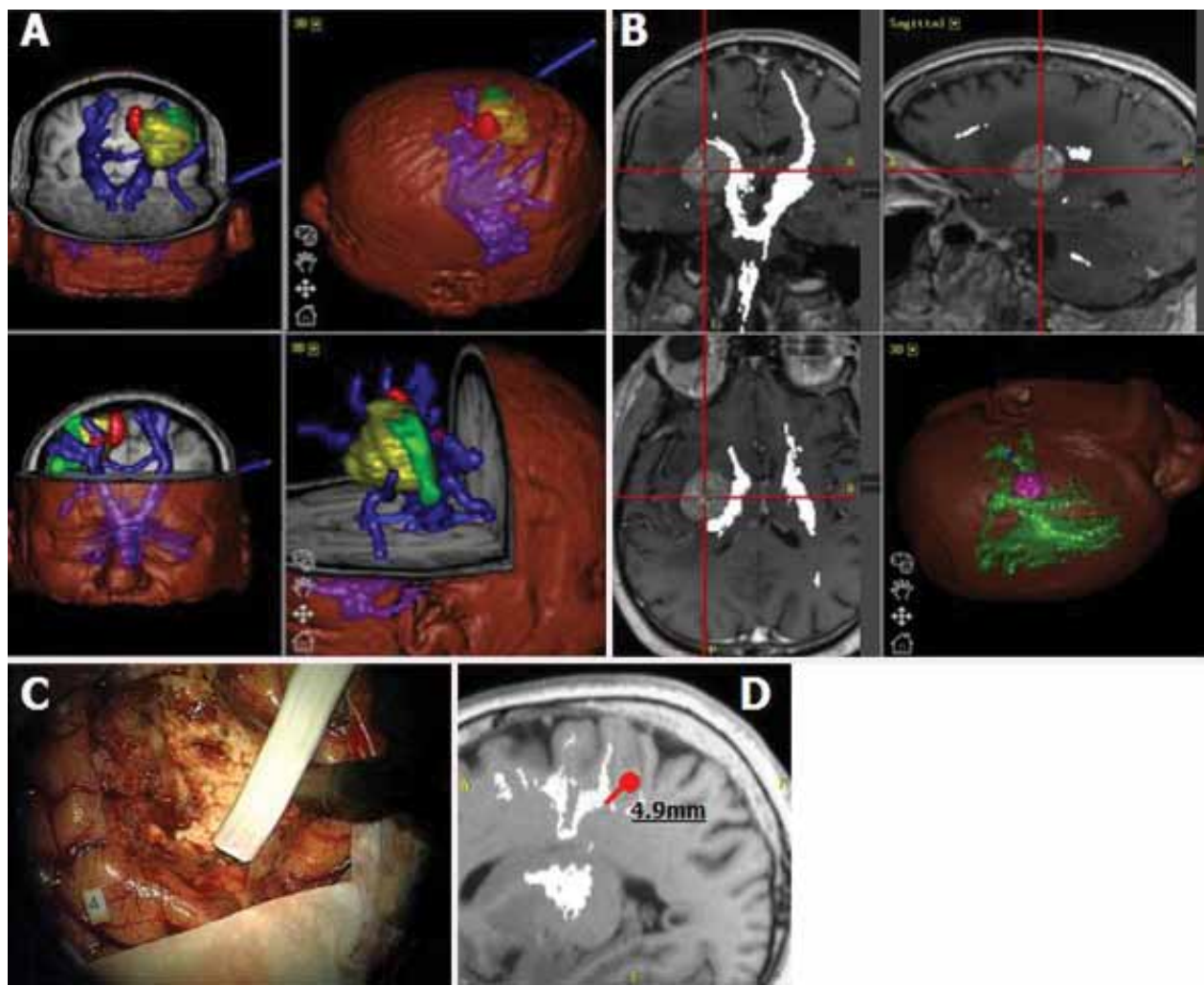

FIG. 2. Intraoperative multimodal subcortical navigation. A: A 3D display of tumor (yellow), hand (green), and foot (red) motor fMR imaging activity and pyramidal tracts (blue) superimposed on a T1 MR imaging anatomical study. B: Pyramidal tracts superimposed on a Gd-enhanced T1-weighted MR imaging study in a patient with a deep-seated metastasis. C: Intraoperative photograph showing an SCS after microsurgical resection of a cortico-subcortical tumor. D: Real-time navigation of a point subjected to sCS with positive motor response (red dot and line), and the measurement of the distance to the nearest corticospinal tract.

were recorded in each case, and the distance between the point of response to the stimulation and the stimulated anatomical tract was calculated on the navigator. We considered it a good correlation if motor responses were obtained when stimulating the cortical or subcortical areas with suspected motor function, and the distances to the navigated corticospinal tract were related between 3.0 and $11.0 \mathrm{~mm}$, according to previous studies ${ }^{4}$ and our own experience.

\section{Surgical Procedure and Follow-Up Data}

All patients underwent surgery after induction of general anesthesia and in the absence of neuromuscular relaxation to allow for intraoperative neurophysiological monitoring. Wide craniotomies were designed, centered on the lesion that had been located with the navigator, including also the eloquent cortical area defined based on intraoperative study. The lesion was resected with the aid of a surgical microscope (Pentero), via a transsulcal approach, and according to standard microsurgical techniques, including the use of an ultrasonic aspirator. We used fluorescence techniques after administration of 5 -aminolevulinic acid in the 8 cases we investigated in which important contrast enhancement and the suspicion of high-grade behavior occurred. The objective of the surgery was the complete resection of the lesion defined in the planning as tumor, with the widest possible margin of tissue removal without causing any new neurological deficits (so-called functional resection). The anesthesia was reversed in the operating room itself or in the recovery room in the first 6 hours after the surgery. Apart from the routine neurological progress evaluations, a neurological assessment was performed according to the protocol at 24 hours and at 30 days after surgery, and any new neurological deficits and the evolution of the previous ones were recorded. Apart from the routine postoperative CT scans obtained for follow-up evaluation of the surgery, an MR imaging session was performed within 72 hours for resection assessment, with the following: axial T1weighted sequences with Gd contrast, axial FLAIR, and DT images. Using these studies, after a new volumetric analysis, a calculation was made of the extent of tumor resection, and 4 groups were defined: total (100\%); subtotal (90-99\%); partial (50-89\%); and suboptimal resection (< $50 \%$ ), according to the classification proposed by Berger et al. ${ }^{2}$ Finally, the data provided by the anatomopathological study were collected.

\section{Results}

In this study, a total of 17 patients were included who underwent surgery for tumors in eloquent motor ar- 
TABLE 1: Preoperative motor deficit, results of SCS and postsurgical focality, and distance between sCS points with a positive response and involved fasciculi on DT imaging navigation in 17 patients who underwent functional microsurgical resection of brain tumors*

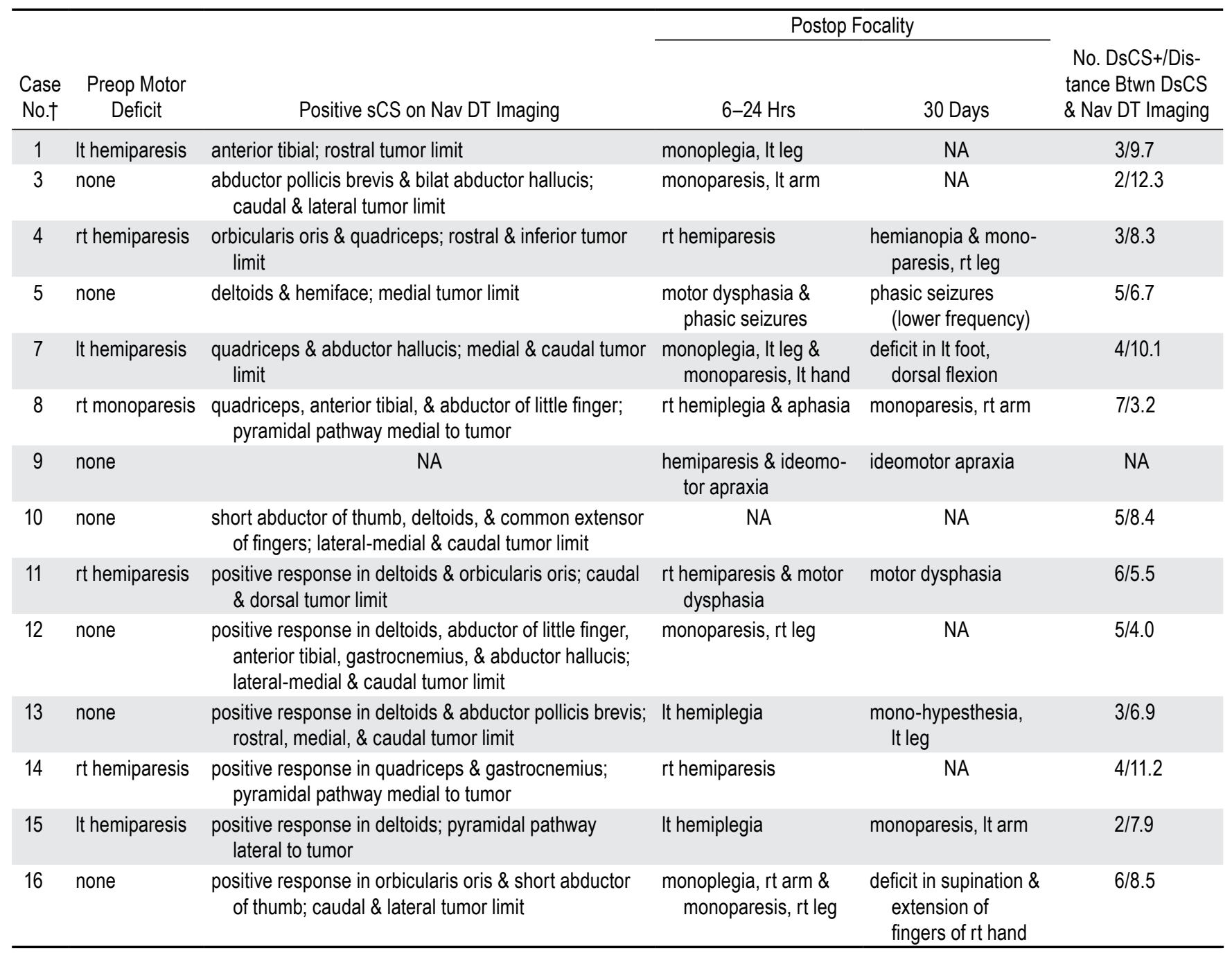

* DsCS+ = positive direct sCS; NA = not applicable; Nav = navigated.

$\dagger$ Cases 2, 6, and 17 were omitted because there was no positive response after sCS.

eas between June 2008 and April 2009 at the Neurosurgery Department of the Hospital Clínico Universitario of Valencia. The series was composed of 7 women and 10 men (1:1.4) with a mean age $( \pm \mathrm{SD})$ of $54 \pm 9.5$ years. The principal clinical presentation was irritative in 11 patients (64.7\%), with contralateral deficit (Table 1) in 5 patients (29.4\%), and in the remaining patient (5.9\%) the presenting symptom was headache refractory to analgesic treatment. All patients received symptomatic, antiepileptic, or corticosteroid treatment before the intervention, with control or improvement of the symptoms. After neurological examination performed preoperatively, a total of $58.8 \%$ of patients had some kind of motor neurological deficit. Two patients had undergone previous surgery for Grade II oligodendroglioma, presenting now with a local recurrence.

In the CT scan obtained without contrast, the lesions were identified in all cases except one as hypointense masses. Hyperintense areas were found in 3 studies, cor- responding to calcifications in 2 cases and intratumoral bleeding in the remaining one. The lesion was identified on all the MR imaging studies, with a pattern of hypointensity in the T1-weighted sequences and of hyperintensity in the T2-weighted sequences and FLAIR, with uptake of Gd in 8 cases and areas suggestive of necrosis in 3 patients. The perfusion and spectroscopy studies showed patterns of high-grade tumor in 10 patients $(58.8 \%)$ and of low-grade tumor in the remaining 7 cases. The mean tumor volume was $27.2 \pm 21 \mathrm{~cm}^{3}$ (range 3.4-72.7 $\mathrm{cm}^{3}$ ). Assessing the anatomical location of the lesions, the tumor was cortical in 12 patients $(70.6 \%$; 3 in the postcentral gyrus, 4 in the precentral gyrus, 2 in the posterior part of the superior frontal gyrus, 1 in the anterior part of the superior frontal gyrus, and 2 in the frontal operculum), and the lesions were subcortical in the 5 remaining cases $(29.4 \%$; 3 in the corona radiata displacing the internal capsule and 2 in the insular white matter). The dis- 
tribution of the lesions according to the classification of Sawaya et al. ${ }^{36}$ was as follows: 7 Grade II lesions (41.2\%) and 10 Grade III lesions (58.8\%). Functional MR imaging was performed in 11 patients, and showed areas of activation over the contralateral precentral area. Positive areas of activation were obtained superimposed over the lesion in 3 cases, all of them low-grade gliomas in the precentral area. The DT MR imaging studies were completed in all cases. Tractography was performed without any difficulties, with segmentation and individualization of the corticospinal pathway in 16 studies and of the superior longitudinal fasciculus in 6 .

The fusion of the anatomical and diagnostic studies, of fMR imaging and tractography, was achieved in all cases with high precision (estimated error $<1.5 \mathrm{~mm}$ ), in 15 cases $(88.2 \%)$ automatically and in the 2 remaining ones by using points to achieve the same level of precision.

\section{Clinical and Imaging Results}

All the lesions were located during surgery and were anatomopathologically confirmed. There was no intraor postoperative death or morbidity related to the study methods or monitoring. The anatomopathological study showed the following lesions: low-grade astrocytoma (3 cases), anaplastic astrocytoma (3), oligoastrocytoma (2), low-grade oligodendroglioma (2), glioblastoma multiforme (4), and metastasis (3).

Twenty-four hours after surgery, there was deficit or irritative neurological focality in 12 patients $(70.6 \%)$. One month after the surgery, deficits were substantially reduced, both quantitatively ( 8 patients, $47.1 \%$ ) and qualitatively, and daily activities were affected in only 1 patient.

The postoperative volumetric analysis showed an extent of tumor resection of $89.1 \pm 14.2 \%$ of the tumor volume calculated in the preoperative studies, with total resection in 8 patients $(47.1 \%)$, subtotal resection in 3 patients (17.6\%), and partial resection in the 6 remaining patients $(35.3 \%)$. The average resection was higher in the tumors located in the frontal operculum, superior frontal gyrus, and corona radiata, but it was lower in the insular regions and pre- and postcentral gyri. Regarding the correlation between the percentage of resection and the Sawaya grades, we found that in the Grade II tumors the extent of resection was $91.9 \%$, whereas in the Grade III tumors the mean extent of resection was $87.1 \%$. According to the nature of the lesion, the maximum grade of resection was found in metastases and oligodendrogliomas, was lower in anaplastic astrocytomas and glioblastomas, and even lower in low-grade astrocytomas.

\section{Operative Anatomofunctional Correlation Between the Motor Cortex and the Corticospinal Pathway}

Using anatomical references, the central sulcus and the precentral gyrus were located with certainty in 11 studies $(64.7 \%)$, whereas in the 6 remaining cases, definition of the central sulcus was impossible because of the distortion due to the mass effect of the tumor. Functional MR imaging always showed activation over the anatomi- cally located precentral gyrus, although in many cases the precision was poor due to activity over the tumor, and in many other cases there were regions of dispersed activation in front of or behind the precentral gyrus. In all cases in which the corticospinal pathway was defined using tractography, there was complete correlation with the location defined in the anatomical imaging studies, namely the precentral gyrus where this tract originates, including the 6 cases in which the lesions had displaced the normal anatomy more or less grossly, which was extremely useful to confirm the anatomical localization of the central sulcus and precentral gyrus. There was also a good correlation with the areas of activation in the fMR imaging in the majority of cases when both studies were fused.

\section{Intraoperative Anatomofunctional Correlation: Electrical Stimulation and Mapping}

In the tumors located in the postcentral, precentral, and superior frontal gyri, a total of 52 direct cortical stimulations were performed in which a positive motor response identifying the primary motor area occurred. We got a positive correlation in $83.7 \%$ between these points recorded in the navigator and the corresponding functional areas identified in the fMR imaging. In 4 cases of tumors adjacent to the central sulcus, it was necessary to use the $\mathrm{N} 20$ wave inversion technique to locate the lesion due to the anatomical distortion produced by the tumor, which made it impossible to recognize with navigation. In 3 of these cases, the location of the central sulcus was consistent with the volumes of corticospinal tract identified with DT imaging. In the remaining case, the central sulcus located with wave inversion was $4 \mathrm{~mm}$ behind the anatomical sulcus identified according to the navigated tractography. Navigating the images obtained by tractography was really useful when resecting lesions close to the corticospinal pathway. After the volumetric resection, we stimulated the tumor bed to identify the subcortical motor tracts and, in 13 patients, a total of 55 sCS procedures were performed, with positive motor response that identified corticospinal tracts coming from the primary motor area (Table 1). In these cases, the average distance between points of response and the location of the tracts in the navigator was $7.3 \pm 3.1 \mathrm{~mm}$ (range $1.8-13.4 \mathrm{~mm}$ ). Given that the CS and sCS parameters have an influence on the response obtained, stimuli of $0.5-$ to $1-\mathrm{msec}$ duration were used with intensities between 2 and $10 \mathrm{~mA}$. The SSEPs were only altered in one case of deep lesion adjacent to the pyramidal pathway in the layer of the corona radiata.

On analyzing the relationship between the neurological focality at 30 days and the distance between the last point with a positive response to subcortical or corticospinal tract stimulation, an inverse relationship can be seen between them both, with an empirical safety margin of 8-10 mm (Fig. 3).

Between 1 and 3 days after the surgery, a functional mobility study was performed in all the patients included in this work. After this first evaluation, an initial functional rehabilitation based on passive movements was performed in 12 of the patients, continuing, when pos- 


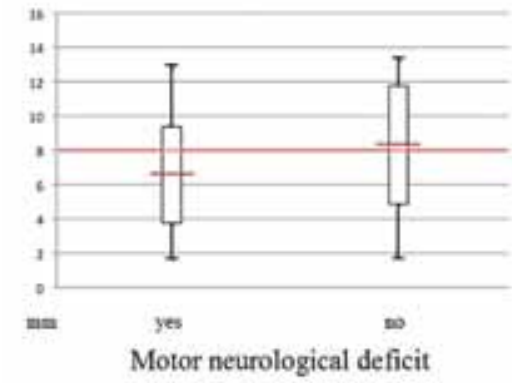

FIG. 3. Boxplot showing the relationship between the presence of focal motor neurological deficits 30 days after surgery and the distance between the point of sCS and the navigated pyramidal tract (short red line).

sible, with active movements to avoid muscle atrophy and improve strength during the first postoperative stage. When the improvement seemed to have peaked, the functional rehabilitation continued with occupational therapy, based on the development of daily activities.

\section{Discussion}

The current recommendation for the treatment of intrinsic brain tumors is radical microsurgical resection of the lesion while maintaining the integrity of neurological function, followed by observation using neuroimaging, or oncological treatment. Given that glial tumors show infiltrating growth regardless of their grade, considering a volumetric resection beyond the limits of the tumor determined by the imaging techniques is necessary. However, this so-called tumor resection limit should only extend to the point where, as a consequence of the tissue resection, deterioration of the neurological function may appear (the functional resection limit).

One of the most critical and eloquent functional activities is motor activity, due to which microsurgical treatment of tumors in this region is particularly interesting. The cortical part of the motor and associated motor areas is composed of the postcentral gyrus, central sulcus, precentral gyrus, superior frontal gyrus, and frontal operculi, and should also include the short subcortical connections between the neighboring gyri ( $\mathrm{U}$-shaped fasciculi) and the long subcortical connections, such as the superior longitudinal fasciculus and particularly the effector corticospinal pathway. ${ }^{3,9,30,34}$ In clinical practice, there is a large number of techniques that can be used to delimit these structures anatomically and functionally and to make surgery on tumors located in these areas safer. ${ }^{1,5,8,15,17,21,23,24,28,29}$

\section{Preoperative and Intraoperative Protocol}

In our study, the cortical areas were anatomically determined with imaging techniques, specifically with 3D reconstructions of MR images. This anatomical identification is usually safe, and although it may be distorted by the underlying pathological entity itself, it is determined preoperatively and checked intraoperatively with navigation, correlating it with the microsurgical field. Functional MR imaging allows a particular function to be preoperatively assigned to a cortical region that is activated as a consequence of the development of an eloquent activity. We have studied motor activity, again with a good anatomical correlation, but also with examinations with contradictory results as a consequence of diffuse activation or activation over the tumor itself. The fMR imaging fusion in the anatomical studies allows this information to be integrated into the intraoperative navigation. . $^{1,10,14,21,27}$ However, there are still doubtful or contradictory cases when making decisions during surgery. In this circumstance, we added neurophysiological monitoring with two techniques in the motor area. First we performed anatomical localization of the central sulcus by using the N20 wave inversion technique, and then we delivered CS with a bipolar electrode..$^{5,8,18,23,27}$ This protocol has led to a great advance in the microsurgical treatment of tumors in motor areas, but only provides information on the cortex, with no data on the anatomical arrangement of the subcortical tracts or on their functional integrity.

Until now, sCS of the resection margins allowed the surgeon to discover the functional integrity of the stimulated fibers, ${ }^{5,8,27}$ but without having a clear idea of the 3D location of the tract in relation to the stimulated point. The possibility of locating the subcortical tracts preoperatively by using DT imaging and tractography has led to a new advance in understanding the anatomical relationships between the lesions and these pathways, because they allow the arrangement of tracts in the white matter to be studied three-dimensionally, voxel by voxel, throughout the entire human brain. ${ }^{3}$ This has been the reason for our interest in incorporating DT imaging into the navigation system, thus discovering preoperatively the anatomical location of the points subjected to sCS, to make decisions on whether to continue with the tissue resection.

\section{Role of Tractography and sCS}

In our experience, adding DT images into the Medtronic StealthStation navigation system has been possible in a simple but very laborious fashion. The preoperative surgery planning work has increased significantly, to get a better anatomical understanding of the subcortical anatomical pathways and of the lesion itself. Formerly, our protocol for preoperative planning and intraoperative monitoring focused on the identification of the lesion, the sulci, the gyri, and specific cortical vascular structures for anatomical reference, and on knowledge of the functionality of eloquent cortical areas. The incorporation of DT imaging and sCS has allowed us to understand the anatomical location of the white matter tracts and to study them functionally, above and beyond the intuition and imagination of the surgeon.

However, the objective of all this effort in terms of human and economic resources is to maximize the resection of lesions in the region and to minimize morbidity. The impression gained after undertaking this work is that the integration of DT imaging into the navigation protocol has substantially increased the extent of tumor resection in motor areas, and that more neurological deficits are produced. In fact, the volumetric analysis of tumor resection is found to be $90 \%$, with total resections in almost half of the series, and at the same time, in the immediate postoperative period nearly all patients have experienced new 
neurological deficits or worsening of preexisting deficits. However, the neurological deficits are totally or partially reversible in the majority of patients, and within 30 days only one patient had presented with problems that made it difficult to conduct daily activities. The literature gives us an awareness of the presence of high rates of neurological focality in the early postoperative period for lesions in eloquent areas, and its subsequent resolution. ${ }^{5,8,10,15}$ This has been related to the fact that anatomical and functional monitoring allows us to work very close to the eloquent areas, in which the inevitable phenomena of traction, heat due to bipolar thermocoagulation, cytotoxic edema, or phenomena of microvascular reorganization would have greater functional repercussions during the immediate postoperative period.

It has already been stated that the processing of DT imaging and its conversion into DICOM and/or Analyze format (which allow for fusion in the navigator with the anatomical and fMR images) consumes a significant amount of time during preoperative planning. However, the information provided by DT imaging allows us to shorten the time spent in the intraoperative process of cortical functional localization with CS and N20 wave inversion to define the sulci for the approach to the tumor and for corticectomy. Also, once the volumetric tumor resection has been undertaken, the anatomical knowledge and the ability to visualize the cortical pathways, and especially the corticospinal pathway by using navigation, reduce the number of sCSs needed to locate the pathways and improve the interpretation of the information obtained to maximize the resection. In this sense, as a consequence of our initial results, a safe distance of 8-10 $\mathrm{mm}$ (Fig. 3) has been established between the point of stimulation at the functional resection limit and the corticospinal tract, which turns out to be similar to that which has been proposed by other authors., 4,529

\section{Limitations of DT Imaging and Tractography}

The wide safety distance established between the point of sCS and the tract may seem excessive after the entire preoperative calculation process. This may be an important point of criticism of the study protocol we used, and may be due to several reasons. The process of obtaining the DT images and subsequent reconstructions to generate 3D images implies a huge number of matrix calculations that may accumulate errors. An important aspect is that the mechanism used to generate the eigenvector (that is, the main direction of the tract included in the seeding area) from the tensor is a user-defined procedure and, consequently, subject to interindividual differences. ${ }^{22}$ The number of fibers that will be included in the tract depends on the anatomical location chosen to generate the tract in question, on the fractional anisotropy values chosen, on the algorithm used, and on the user-defined area. Also, consideration has to be given intraoperatively to the error introduced into the navigation data by the shifting of brain structures after the tumor resection, which includes the phenomenon of expansion generated by the recovery of space lost due to the growth of the tumor, edema due to manipulation, and relative pressure changes. The consequence is that the reexpansion makes the white matter move closer to the center of the resection cavity generated, shortening the distances between the point of sCS and the real location of the stimulated eloquent tract.

After analyzing our results during the sCS, the conclusion we reached is congruent with the results obtained by other authors. The distance between the motor response-stimulated point and the navigated corticospinal fascicle implicated on the transmission of that movement has always been positive in our series $(+1.8$ to $+13.4 \mathrm{~mm})$. This fact means that we have positive responses in tissue localized closer to the center of the resection cavity than to the "real fascicle." If we had only analyzed this fact, we could have concluded that we were leaving some tumor tissue surrounding the corticospinal tract and achieving lower extents of resection. However, when we analyzed the postoperative MR imaging studies, the extent of resection was nearly $90 \%$ of the tumor volume, a fact that leads us to affirm that the shifting of these brain structures was always directed to the center of the resection cavity. This phenomenon of tissue reexpansion during tumor resection has been extensively studied by Archip et al. ${ }^{1}$ and Nimsky and colleagues, ${ }^{29,30}$ who demonstrated using intraoperative MR imaging and DT imaging with tractography of the corticospinal pathway, that while the resection cavity was filled with neighboring parenchyma due to the phenomenon of reexpansion, the tracts of the pyramidal pathway moved closer to the center of the cavity. After our experience, we think it could be really interesting to assess the phenomenon of "growth" of the subcortical pathways affected by surrounding tumors during the surgical decompression, and to analyze the possible responses with the aid of sCS at lower intensities and at some distance away from the fascicles.

In the absence of this sophisticated method of intraoperative control, the combination of the data provided by preoperative DT imaging and intraoperative SCS can be correctly interpreted by introducing a correction factor to compensate for the shift. Thus, Nimsky et al. ${ }^{29}$ propose adding a safety margin to the volume generated by each tract that, based on their own experience, they calculate to be between 5 and $7 \mathrm{~mm}$. This can be determined preoperatively by using fractional anisotropy values $<0.2$, or simply by adding a fictitious volume to that generated after the primary calculation.

\section{Conclusions}

The integration of pre- and intraoperative anatomical and functional studies allows for functional resection that significantly widens the extent of resection in lesions in relevant eloquent areas. Navigation allows us to integrate and understand the correlation between the preoperative data and the intraoperative findings. The cortical functional motor areas are anatomically and functionally defined in the preoperative period by using MR and $\mathrm{fMR}$ imaging studies, and the subcortical functional motor areas are defined with DT imaging and the generation of tractography from the DT images, whereas intraoperative confirmation is achieved using CS and the N20 wave inversion study for the cortical areas and SCS for the subcortical areas. Microsurgical treatment guided by navigation 
and with the aid of the studies described allows for mean tumor resections of $90 \%$ in lesions located in cortical and subcortical eloquent motor areas, with high neurological morbidity in the immediate postoperative period, which is significantly reduced both quantitatively and qualitatively within 4 weeks. The ongoing studies should define safety margins for functional resection that take the brain shift into consideration. Finally, the benefit of these protocols for disease-free interval, recurrence, or ultimate patient survival remains to be defined.

\section{Disclosure}

The authors report no conflict of interest concerning the materials or methods used in this study or the findings specified in this paper.

Author contributions to the study and manuscript preparation include the following. Conception and design: JM González-Darder. Acquisition of data: V Cortés. Analysis and interpretation of data: P González-López, F Talamantes, V Quilis, G García-March, P Roldán.

\section{References}

1. Archip N, Clatz O, Whalen S, Kacher D, Fedorov A, Kot A, et al: Non-rigid alignment of pre-operative MRI, fMRI, and DT-MRI with intra-operative MRI for enhanced visualization and navigation in image-guided neurosurgery. Neuroimage 35:609-624, 2007

2. Berger MS, Deliganis AV, Dobbins J, Keles GE: The effect of extent of resection on recurrence in patients with low grade cerebral hemisphere gliomas. Cancer 74:1784-1791, 1994

3. Berger MS, Hadjipanayis CG: Surgery of intrinsic cerebral tumors. Neurosurgery 61 (1 Suppl):279-305, 2007

4. Berman JI, Berger MS, Chung SW, Nagarajan SS, Henry RG: Accuracy of diffusion tensor magnetic resonance imaging tractography assessed using intraoperative subcortical stimulation mapping and magnetic source imaging. J Neurosurg 107:488-494, 2007

5. Brell M, Conesa G, Acebes JJ: [Intraoperative cortical mapping in the surgical resection of low-grade gliomas located in eloquent areas.] Neurocirugia 14:491-503, 2003 (Sp)

6. Bürgel U, Amunts K, Hoemke L, Mohlberg H, Gilsbach JM, Zilles K: White matter fiber tracts of the human brain: threedimensional mapping at microscopic resolution, topography and intersubject variability. Neuroimage 29:1092-1105, 2006

7. Claes A, Idema AJ, Wesseling P: Diffuse glioma growth: a guerilla war. Acta Neuropathol 114:443-458, 2007

8. Duffau H, Peggy Gatignol ST, Mandonnet E, Capelle L, Taillandier L: Intraoperative subcortical stimulation mapping of language pathways in a consecutive series of 115 patients with Grade II glioma in the left dominant hemisphere. J Neurosurg 109:461-471, 2008

9. Fernández-Miranda JC, Rhoton AL Jr, Alvarez-Linera J, Kakizawa Y, Choi C, de Oliveira EP: Three-dimensional microsurgical and tractographic anatomy of the white matter of the human brain. Neurosurgery 62 (6 Suppl 3):989-1028, 2008

10. Fontaine D, Capelle L, Duffau H: Somatotopy of the supplementary motor area: evidence from correlation of the extent of surgical resection with the clinical patterns of deficit. Neurosurgery 50:297-305, 2002

11. Giese A, Bjerkvig R, Berens ME, Westphal M: Cost of migration: invasion of malignant gliomas and implications for treatment. J Clin Oncol 21:1624-1636, 2003

12. Giese A, Kluwe L, Laube B, Meissner H, Berens ME, Westphal M: Migration of human glioma cells on myelin. Neurosurgery 38:755-764, 1996
13. Giese A, Loo MA, Rief MD, Tran N, Berens ME: Substrates for astrocytoma invasion. Neurosurgery 37:294-302, 1995

14. Hashiguchi M, Morioka T, Yoshida F, Miyagi Y, Shono T, Nagata $\mathrm{S}$, et al: Usefulness of functional neuronavigation system integrated with functional MRI and corticospinal tractography. Clin Neurophysiol 118:e189-e202, 2007

15. Hentschel SJ, Sawaya R: Optimizing outcomes with maximal surgical resection of malignant gliomas. Cancer Control 10:109-114, 2003

16. Hwang JH, Smith CA, Salhia B, Rutka JT: The role of fascin in the migration and invasiveness of malignant glioma cells. Neoplasia 10:149-159, 2008

17. Kamada K, Todo T, Masutani Y, Aoki S, Ino K, Morita A, et al: Visualization of the frontotemporal language fibers by tractography combined with functional magnetic resonance imaging and magnetoencephalography. J Neurosurg 106:90-98, 2007

18. Kamada K, Todo T, Morita A, Masutani Y, Aoki S, Ino K, et al: Functional monitoring for visual pathway using real-time visual evoked potentials and optic-radiation tractography. Neurosurgery 57 (1 Suppl):121-127, 2005

19. Keles GE, Chang EF, Lamborn KR, Tihan T, Chang CJ, Chang $\mathrm{SM}$, et al: Volumetric extent of resection and residual contrast enhancement on initial surgery as predictors of outcome in adult patients with hemispheric anaplastic astrocytoma. J Neurosurg 105:34-40, 2006

20. Keles GE, Lamborn KR, Berger MS: Low-grade hemispheric gliomas in adults: a critical review of extent of resection as a factor influencing outcome. J Neurosurg 95:735-745, 2001

21. Kim P, Singh M: Functional magnetic resonance imaging for brain mapping in neurosurgery. Neurosurg Focus 15(1):1-7, 2003

22. Kinoshita M, Yamada K, Hashimoto N, Kato A, Izumoto S, Baba T, et al: Fiber-tracking does not accurately estimate size of fiber bundle in pathological condition: initial neurosurgical experience using neuronavigation and subcortical white matter stimulation. Neuroimage 25:424-429, 2005

23. Kral T, Kurthen M, Schramm J, Urbach H, Meyer B: Stimulation mapping via implanted grid electrodes prior to surgery for gliomas in highly eloquent cortex. Neurosurgery 58 (1 Suppl):ONS36-ONS 43, 2006

24. Mäkelä JP, Forss N, Jääskeläinen J, Kirveskari E, Korvenoja A, Paetau R: Magnetoencephalography in neurosurgery. Neurosurgery 59:493-511, 2006

25. McGirt MJ, Chaichana KL, Attenello FJ, Weingart JD, Than $\mathrm{K}$, Burger PC, et al: Extent of surgical resection is independently associated with survival in patients with hemispheric infiltrating low-grade gliomas. Neurosurgery 63:700-708, 2008

26. McGirt MJ, Chaichana KL, Gathinji M, Attenello FJ, Than K, Olivi A, et al: Independent association of extent of resection with survival in patients with malignant brain astrocytoma. J Neurosurg 110:156-162, 2009

27. Mikuni N, Okada T, Enatsu R, Miki Y, Hanakawa T, Urayama $\mathrm{S}$, et al: Clinical impact of integrated functional neuronavigation and subcortical electrical stimulation to preserve motor function during resection of brain tumors. J Neurosurg 106:593-598, 2007

28. Mikuni N, Okada T, Nishida N, Taki J, Enatsu R, Ikeda A, et al: Comparison between motor evoked potential recording and fiber tracking for estimating pyramidal tracts near brain tumors. J Neurosurg 106:128-133, 2007

29. Nimsky C, Ganslandt O, Hastreiter P, Wang R, Benner T, Sorensen $\mathrm{AG}$, et al: Preoperative and intraoperative diffusion tensor imaging-based fiber tracking in glioma surgery. Neurosurgery 56:130-138, 2005

30. Nimsky C, Ganslandt O, Merhof D, Sorensen AG, Fahlbusch $\mathrm{R}$ : Intraoperative visualization of the pyramidal tract by 
diffusion-tensor-imaging-based fiber tracking. Neuroimage 30:1219-1229, 2006

31. Pesudo JV, González-Darder JM, Feliu R, Belloch V, Vera J, Gil JL: [Assessment of the degree of resection of high grade supratentorial gliomas with early postoperative magnetic resonance.] Neurocirugia 12:43-50, 2001 (Sp)

32. Price SJ, Jena R, Burnet NG, Hutchinson PJ, Dean AF, Peña A, et al: Improved delineation of glioma margins and regions of infiltration with the use of diffusion tensor imaging: an image-guided biopsy study. AJNR Am J Neuroradiol 27:19691974, 2006

33. Provenzale JM, McGraw P, Mhatre P, Guo AC, Delong D: Peritumoral brain regions in gliomas and meningiomas: investigation with isotropic diffusion-weighted MR imaging and diffusion-tensor MR imaging. Radiology 232:451-460, 2004

34. Rhoton AL Jr: The cerebrum. Anatomy. Neurosurgery 61 (1 Suppl):37-119, 2007

35. Sanai N, Berger MS: Glioma extent of resection and its impact on patient outcome. Neurosurgery 62:753-766, 2008

36. Sawaya R, Hammoud M, Schoppa D, Hess KR, Wu SZ, Shi WM, et al: Neurosurgical outcomes in a modern series of 400 craniotomies for treatment of parenchymal tumors. Neurosurgery 42:1044-1056, 1998

37. Shaw EG, Berkey B, Coons SW, Bullard D, Brachman D, Buckner JC, et al: Recurrence following neurosurgeon-determined gross-total resection of adult supratentorial low-grade glioma: results of a prospective clinical trial. J Neurosurg 109:835-841, 2008

38. Stummer W, Stocker S, Wagner S, Stepp H, Fritsch C, Goetz C, et al: Intraoperative detection of malignant gliomas by 5 -aminolevulinic acid-induced porphyrin fluorescence. Neurosurgery 42:518-526, 1998

39. Tanaka Y, Nariai T, Momose T, Aoyagi M, Maehara T, Tomori $\mathrm{T}$, et al: Glioma surgery using a multimodal navigation system with integrated metabolic images. J Neurosurg 110:163-172, 2009

Manuscript submitted October 11, 2009.

Accepted November 30, 2009.

Address correspondence to: José M González-Darder, M.D., Department of Neurosurgery, Hospital Clínico Universitario, Avenida Blasco Ibáñez, 17, 46010 Valencia, Spain. email: gonzalez_jos@gva.es. 